\title{
Effect of endometrial thickness and embryo quality on live-birth rate of fresh IVF/ICSI cycles: a retrospective cohort study
}

Hong Lv ${ }^{1,2+}$, Xiuzhu Li ${ }^{1 \dagger}$, Jiangbo Du ${ }^{1,2+}$, Xiufeng Ling ${ }^{3}$, Feiyang Diao ${ }^{4}$, Qun Lu², Shiyao Tao ${ }^{1,2}$, Lei Huang ${ }^{1,2}$,

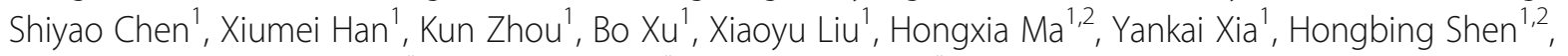
Zhibin $\mathrm{Hu}^{1,2}$, Guangfu Jin ${ }^{1,2^{*}}$ (D), Yichun Guan ${ }^{5^{*}}$ and Xinru Wang ${ }^{1 *}$

\begin{abstract}
Background: Successful implantation and delivery require both the functional embryo and receptive endometrium in assisted reproductive technology (ART) cycles. However, little is known about embryo-endometrial interaction on live-birth. We aimed to investigate the independent effect and interaction of endometrial thickness (EMT) and embryo quality on live-birth in fresh embryo transfer (ET) cycles.

Methods: We conducted a retrospective cohort study including 15,012 ART cycles between 2013 and 2016 in three centers in China. Poisson regression with generalized estimating equations was employed to calculate relative risks (RRs) and 95\% confidence intervals (Cls). We estimated the interaction of embryo quality and EMT on live-birth rate (LBR).

Results: The LBR per cycle was $42.8 \%$ overall. LBR increased with increasing EMT and reached a plateau (50.6 to 54.2\%) when EMT was $11 \mathrm{~mm}$ or thicker. Embryo quality represented by cumulative score was associated with LBR independently of number of embryos transferred and EMT. LBR was not increased with thicker EMT when only Q1 cleavage-stage embryo transferred (aRR 0.95, 95\%Cl 0.61-1.46). LBR was not increased significantly with thicker EMT with transfer of two good-quality cleavage-stage embryos and any blastocyst combination except Q1 group. There was significant interaction between EMT and embryo quality on LBR for cleavage-stage ETs ( $P=0.023)$.

Conclusions: This study demonstrated the nonlinear EMT-LBR association and the EMT cut-off value of $11 \mathrm{~mm}$ which may be of more clinical significance for predicting live-birth. Embryo quality is an independent prognostic tool for LBR. Our finding of significant embryo-endometrial interaction indicates combination of EMT and embryos quality might improve the prognostic value in clinical practice for live-birth in patients undergoing transfer of 1-2 fresh cleavage-stage embryos.
\end{abstract}

Keywords: Endometrial thickness, Embryo quality, IVF/ICSI, Live-birth rate per cycle, Interaction

\footnotetext{
* Correspondence: guangfujin@njmu.edu.cn; lisamayguan@126.com; xrwang@njmu.edu.cn

${ }^{\dagger}$ Hong LV, Xiuzhu Li and Jiangbo Du contributed equally to this work.

${ }^{1}$ State Key Laboratory of Reproductive Medicine, Nanjing Medical University, Nanjing 211166, China

${ }^{5}$ Department of Reproduction, Henan Medical Maternity and Child Health Care Hospital, Henan 450052, China

Full list of author information is available at the end of the article
}

(c) The Author(s). 2020 Open Access This article is licensed under a Creative Commons Attribution 4.0 International License, which permits use, sharing, adaptation, distribution and reproduction in any medium or format, as long as you give appropriate credit to the original author(s) and the source, provide a link to the Creative Commons licence, and indicate if changes were made. The images or other third party material in this article are included in the article's Creative Commons licence, unless indicated otherwise in a credit line to the material. If material is not included in the article's Creative Commons licence and your intended use is not permitted by statutory regulation or exceeds the permitted use, you will need to obtain permission directly from the copyright holder. To view a copy of this licence, visit http://creativecommons.org/licenses/by/4.0/ The Creative Commons Public Domain Dedication waiver (http://creativecommons.org/publicdomain/zero/1.0/) applies to the data made available in this article, unless otherwise stated in a credit line to the data. 


\section{Background}

Over the past decades, advances in clinical and laboratory techniques have substantially improved pregnancy rates and live-birth rates for assisted reproductive technology (ART). However, as reported by the Centers for Disease Control in the United States by the end of 2012, only $38.1 \%$ of all transfer cycles could result in live births [1]. Maternal age has been proved as the most predominant predictor for successful ART outcome [2]. During the process of ART treatment, the endometrial receptivity, and the number and quality of embryos transferred are also regarded as the key determinants for the outcome of in vitro fertilization (IVF)/intracytoplasmic sperm injection (ICSI) cycles [3-5].

As an indicator of endometrial receptivity, endometrial thickness (EMT) on the day of human chorionic gonadotropin (hCG) administration has been reported and reaffirmed as a potential prognostic tool for ART outcomes in multiple studies [6-8], despite significant advancements of ultrasonic [9], immunologic [10] and molecular [11] markers for endometrial receptivity. Although it remains a controversial issue, it has been widely suggested that a thin endometrium is associated with lower chance to conceive after IVF/ICSI, with cut-off values of EMT varying 7-9 $\mathrm{mm}$ in earlier studies [6, 12]. Also, several investigators have addressed the question whether there is a threshold value for thickness above which implantation is unlikely to occur, but the conclusion is contradictory $[13,14]$. In a one-center study of 3350 IVF cycles, the non-linear association of EMT with live-birth was shown and required verification [7].

The number and quality of embryos transferred are the most important factors for IVF/ICSI outcome [15, 16]. Embryo morphological features assessed by optical microscopy are routine criteria applicable for embryo quality evaluation [17]. Several classification systems for embryo quality have been developed, and embryos have been graded into three categories: Good-quality, Fairquality and Poor-quality $[18,19]$. Evidences support the positive role of good-quality embryo for a better outcome of IVF [20, 21]. In addition, although two embryos transferred in one cycle have higher probability of livebirth than one embryo, multiple pregnancy may lead to the elevation of maternal and neonatal risks [22]. Therefore, to determine the optimal number and quality of embryos to transfer, cumulative embryo score (CES) and other embryo scoring methods based on the number of good-quality embryos transferred have been proposed $[15,23,24]$ to predict pregnancy outcomes. However, the efficacy of these embryo scoring systems needs more optimization, because the association between quality of different embryo combinations and live-birth was not taken into consideration.
Successful pregnancy depends upon implantation, a complex process involving reciprocal interactions between the receptive endometrium and functional embryo in ART cycles. However, little is known about this embryo-endometrial interaction on live-birth. Thus, we hypothesized that the combination of EMT and embryo quality may improve the prognostic value in clinical practice for live-birth. Therefore, this multicenter study was designed to assess the association of EMT, embryo quality with live-birth independently and their interaction on live-birth based on a large scale of IVF/ICSI cycles.

\section{Methods}

\section{Study population}

A retrospective cohort study was conducted in three reproductive centers in China, including the First Affiliated Hospital with Nanjing Medical University (the First Affiliated Hospital of NMU), the Affiliated Nanjing Maternity and Child Health Hospital of Nanjing Medical University (NMU Affiliated Maternity Hospital) and Henan Medical Maternity and Child Health Care Hospital. All fresh IVF/ICSI embryo transfer (ET) cycles $(N=$ 17,540) between January 2013 and December 2016 were included. Exclusion criteria were as follows: 1) frozen oocyte or sperm, 2) preimplantation genetic diagnosis (PGD) or preimplantation genetic screening (PGS) or in vitro maturation (IVM), 3) D1 embryo transferred, 4) uterine or endometrial anomalies, 5) three or more embryos transferred, 6) cycles with missing data of EMT, number of embryos transferred and embryo grading. Finally, a total of 15,012 ET cycles were included for analysis. The flowchart is described in detail in Fig. 1.

\section{Baseline characteristics}

Baseline characteristics of couples including demographics and fertility characteristics were obtained from the electronic medical records of reproductive centers. Demographics included maternal age and body mass index (BMI). Fertility characteristics included basal serum levels of follicle stimulating hormone (FSH), luteinizing hormone $(\mathrm{LH})$, testosterone $(\mathrm{T})$ and prolactin (PRL), duration of infertility, infertility type and causes of infertility. Primary infertility was defined as the inability to achieve a clinical pregnancy after 12 months of unprotected and regular sexual intercourse when a woman has never conceived, while secondary infertility was the incapability to conceive in a couple who have had at least one successful clinical pregnancy previously. The cause of infertility was categorized as male factor, tubal factor, anovulation factor and unexplained factor. 


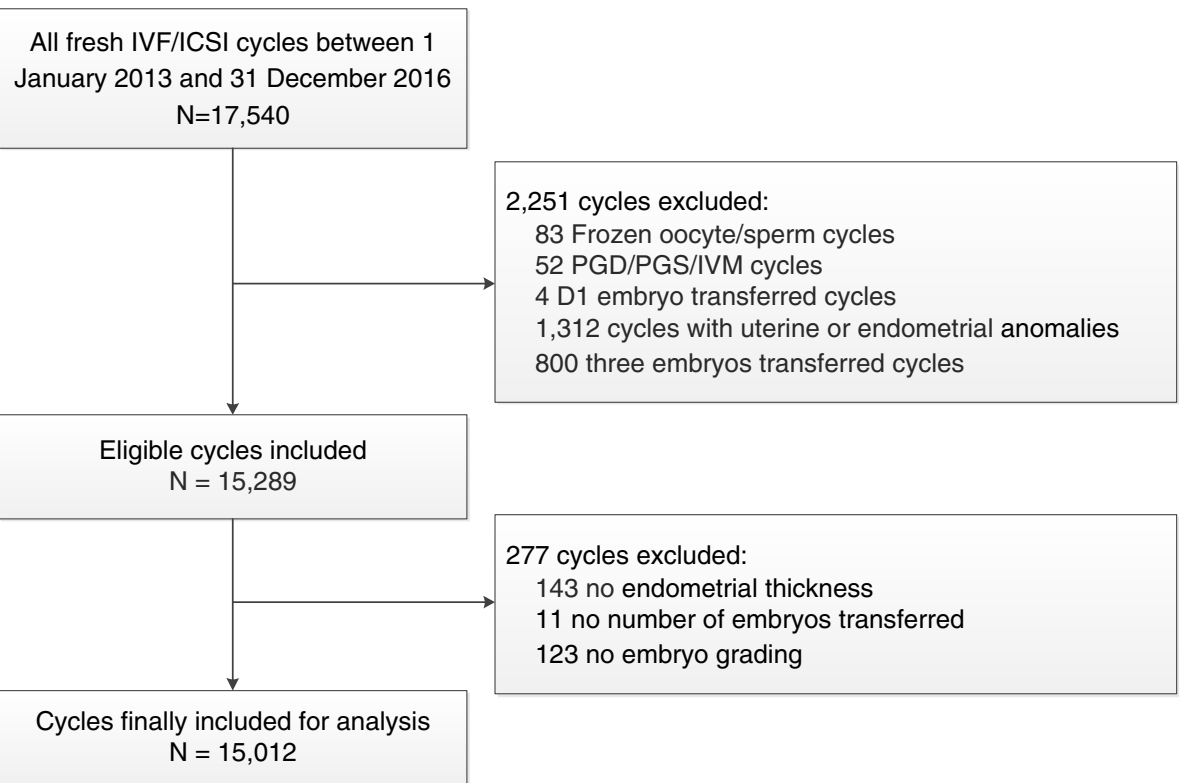

Fig. 1 Flowchart of included and excluded cycles

\section{Treatment characteristics}

The parameters related to treatment included gonadotropin (Gn) dosage and duration, cycle protocols, fertilization methods (IVF, ICSI, or mixed IVF ICSI), serum level of estradiol (E2) and progesterone (P) on hCG day, year of transfer (2013, 2014, 2015 or 2016), stage of embryos transferred (cleavage-stage embryo or blastocyst), number of embryos transferred (1 or 2 ) and oocytes retrieved. Controlled ovarian hyperstimulation $(\mathrm{COH})$ protocols were divided into six categories, including long agonist protocol, short agonist protocol, antagonist protocol, minimal-stimulation protocol, ultralong protocol and other protocol [25].

\section{Endometrial thickness and embryo quality}

EMT was measured in the midsagittal plane of the uterine body on the day of HCG administration. To facilitate the application of the results into clinical practice, we assigned EMT as regular 2-mm-intervalled categories (< $7 \mathrm{~mm}, \geq 7$ to $<9 \mathrm{~mm}, \geq 9$ to $<11 \mathrm{~mm}, \geq 11$ to $<13 \mathrm{~mm}, \geq 13$ to $<15 \mathrm{~mm}, \geq 15$ to $<17 \mathrm{~mm}, \geq 17 \mathrm{~mm}$ ). Cleavage-stage embryos were graded in three categories (Good, Fair and Poor) according to ASEBIR embryo assessment criteria [18], taking into account seven parameters (Day, Cell number, Fragmentation, Symmetry, Multi-nucleation, Vacuoles, Zona pellucida). In a combination of the stage of embryo development and the morphologic grade of the inner cell mass and trophectoderm, blastocysts were divided into three groups based on consensus scoring system for blastocysts: Good (1-6AA, 3-6AB, 3-6BA), Fair (3-6BB, 3-6 AC, 3-6CA, 1-2AB, 1-2BA), and Poor (1-6 $\mathrm{BC}, 1-6 \mathrm{CB}, 1-6 \mathrm{CC}, 1-2 \mathrm{BB})$ [21]. With reference to cumulative embryo score (CES), we derived cumulative score by adding the individual quality score of all the embryos transferred (Poor $=1$ point, Fair $=2$ points, Good $=3$ points) to determine the current group of cleavage-stage embryo quality $(\mathrm{Q} 1=1$ point, $\mathrm{Q} 2=2$ points, $\mathrm{Q} 3=3$ points, $\mathrm{Q} 4=4$ points, $\mathrm{Q} 5=5$ points, $\mathrm{Q} 6=6$ points). In consideration of the limited blastocyst cycles, we finalized the current group of blastocyst embryo quality (Q1 $=1$ point, $\mathrm{Q} 2=2$ points, Q3 $=3$ points, $\mathrm{Q} 4=4 / 5 / 6$ points). We have confirmed the efficiency of CES respectively for both cleavage-stage embryos and blastocysts by comparing the differences of LBR per cycle after transfers of different embryo combinations when 'Good' was set as the reference group in Additional file 1: Table S1.

\section{Outcomes}

Live-birth was defined as the birth of at least one live born neonate beyond 24 weeks of gestational age. The live-birth rate (LBR) per cycle was defined as the rate of achieving a live birth from a fresh ET cycle.

\section{Statistical analysis}

Differences between demographics and clinical characteristics of patients undergoing ART treatment were calculated by t-test or Kruskal-Wallis test as appropriate for continuous variables and chi-square test for categorical variables. Poisson regression with generalized estimating equations was employed to calculate relative risks (RRs) and 95\% confidence intervals (CIs) to estimate the association of live-birth after IVF/ICSI treatment with embryo score and EMT. To adjust for the possible dependence in outcome introduced by repeated 
cycles in the same couple, we constructed models with couple's identification number as a cluster. For the association between EMT and live-birth, we selected group EMT of $\geq 11$ to $<13 \mathrm{~mm}$ as the reference. We adjusted the multiple models for potential confounders including maternal age (continuous variable), maternal BMI (continuous variable), basal FSH (continuous variable), basal $\mathrm{LH}$ (continuous variable), basal $\mathrm{T}$ (continuous variable), basal PRL (continuous variable), duration of infertility (continuous variable), infertility type (primary/secondary), male factor of infertility (yes/no), anovulation factor of infertility (yes/no), Gn dosage (continuous variable), Gn duration (continuous variable), cycle protocols (long agonist protocol/short agonist protocol/antagonist protocol/minimal-stimulation protocol/ultra-long proto$\mathrm{col} /$ other protocol), E2 on hCG day (continuous variable), year of transfer (2013/2014/2015/2016), oocytes retrieved (continuous variable), number of embryos transferred (1/2), embryo quality as well as location of fertility centers to control heterogeneity of multicenter. To test whether the association of embryo quality with live-birth was independent of number of embryos transferred, we ran separate models with and without number of embryos transferred as a sensitivity analysis. Specific subgroup analyses on embryo stage were performed to estimate its potential influence on these relationships. Furthermore, we estimated the multiplicative interaction of EMT and embryo quality on LBR per cycle. Given the heterogeneity in the stimulation protocols for $\mathrm{COH}$, sensitivity analysis was also performed by restricting the analysis to long agonist protocol, the most common type of protocol. Data analysis was conducted using $\mathrm{R}$ software (Version 3.3.3, 2017-03-06; R Foundation for Statistical Computing, http://www.cran.r-project.org/). Statistical significance was interpreted as $P$-value $<0.05$.

\section{Results}

In total, 15,012 IVF/ICSI cycles from 13,909 patients were analyzed during the study period. Table 1 summarized basic characteristics of the patients undergoing ART treatment who were 31.23 years old on average. The LBR per cycle was $42.8 \%$ overall. LBR per cycle was higher in patients with primary infertility compared with secondary type $(45.4 \%$ vs. $39.5 \%)$. Patients receiving ultra-long protocol showed highest LBR per cycle $(52.3 \%)$ in all $\mathrm{COH}$ protocols. LBR per cycle elevated from 39.5 to $46.2 \%$ with year of transfer. $94.8 \%$ of cycles transferred cleavage-stage embryos while only $5.2 \%$ transferred blastocysts. $71.7 \%$ of cycles had two embryos transferred while $28.3 \%$ had a single ET, and after comparisons, LBR per cycle was significantly higher for two ETs than only one embryo (48.2\% vs. $29.1 \%, P<0.001)$.

\section{Effect of EMT on LBR of fresh IVF/ICSI cycles}

The LBR increased with increasing EMT on hCG day as either a continuous variable or ordinal categories after adjusting the confounders (both $P_{\text {trend }}<0.001$, data not shown). As shown in Fig. 2, compared with EMT $\geq 11$ to $<13 \mathrm{~mm}$, EMT less than $7 \mathrm{~mm}, \geq 7$ to $<9 \mathrm{~mm}, \geq 9$ to $<11$ $\mathrm{mm}$ were associated with decreased LBR of 37,22 and $9 \%$, respectively. LBR per cycle reached a plateau (50.6 to $54.2 \%$ ), when EMT was $11 \mathrm{~mm}$ or thicker. No differences were noted when EMT was $\geq 13$ to $<15 \mathrm{~mm}$ (aRR $1.03,95 \% \mathrm{CI} 0.97-1.09), \geq 15$ to $<17 \mathrm{~mm}(1.05,0.95-1.15)$ and more than $17 \mathrm{~mm}(0.98,0.81-1.18)$ in the adjusted model. Hence, the cut-off value for EMT was set as 11 $\mathrm{mm}$. We observed that women with EMT $\geq 11 \mathrm{~mm}$ enjoyed an increased LBR compared with EMT $<11 \mathrm{~mm}$, with aRR of 1.18 (95\%CI 1.14-1.23).

\section{Effect of embryo quality on LBR of fresh IVF/ICSI cycles}

In Table 2, we observed that LBR significantly increased as cleavage-stage embryo quality elevated $\left(P_{\text {for }}\right.$ trend $<$ 0.001 ). In sensitivity analysis, similar results were observed when the analysis was adjusted for the number of embryos transferred. Additional file 1: Table S2 also showed LBR significantly increased as blastocyst quality elevated $\left(P_{\text {for }}\right.$ trend $\left.<0.001\right)$ with and without number of embryos transferred. The association of embryo quality with live-birth remained unchanged after adjustment for EMT regardless of cleavage-stage embryo or blastocyst transfer.

\section{Interaction of EMT and embryo quality on live-birth}

We also analyzed the relationship between cleavagestage embryo quality and live-birth stratified by EMT and the relationship between EMT and live-birth stratified by cleavage-stage embryo quality (Table 3 ). We found LBR increased in higher embryo quality group than Q1 (poor) group in patients with EMT $<11 \mathrm{~mm}$ or EMT $\geq 11 \mathrm{~mm}$. Stratified by cleavage-stage embryo quality, we found LBR did not increase with thicker EMT when only Q1 embryo (aRR $0.95,95 \%$ CI $0.61-1.46$ ) or Q6 embryos (aRR 1.12, 95\%CI 0.98-1.27) were for transfer, but LBR did increase with thicker EMT in other groups of embryo quality. Test for multiplicative interaction was significant $\left(P_{\text {for interaction }}=0.023\right)$. While for blastocyst transfers, we found LBR in Q3 and Q4 embryo quality group significantly increased compared with Q1 group when EMT was $\geq 11 \mathrm{~mm}$ in Additional file 1: Table S3. When stratified by embryo quality, LBR did not increase significantly with thicker EMT in groups of embryo quality except Q1 group. Statistical interaction between EMT and blastocyst embryo quality on livebirth was not significant $(P=0.860)$. The sensitivity analysis restricted to long agonist protocol was shown in Additional file 1: Table S4. No remarkable changes in 
Table 1 Basic characteristics of the patients undergoing IVF/ICSI treatment

\begin{tabular}{|c|c|c|}
\hline Characteristics & $\begin{array}{l}\text { All cycles } \\
(\boldsymbol{n}=15,012)\end{array}$ & $\begin{array}{l}\text { LBR per } \\
\text { cycle }(\%\end{array}$ \\
\hline \multicolumn{3}{|l|}{ Demographics } \\
\hline Maternal age, years, mean $(S D)^{*}$ & $31.23(5.29)$ & \\
\hline Maternal BMI, kg/m², mean (SD) & $22.65(3.26)$ & \\
\hline \multicolumn{3}{|l|}{ Fertility characteristics } \\
\hline Basal FSH, IU/L, mean $(S D)^{*}$ & $7.46(6.23)$ & \\
\hline Basal LH, IU/L, mean $(S D)^{*}$ & $4.58(6.33)$ & \\
\hline Basal $\mathrm{T}, \mathrm{ng} / \mathrm{ml}$, mean $(\mathrm{SD})^{*}$ & $10.21(16.44)$ & \\
\hline Basal PRL, ng/ml, mean (SD) ${ }^{*}$ & 23.98 (222.33) & \\
\hline $\begin{array}{l}\text { Duration of infertility, } \\
\text { years, mean (SD) }\end{array}$ & $3.97(3.20)$ & \\
\hline \multicolumn{3}{|l|}{ Infertility type, $\mathrm{n}(\%)^{*}$} \\
\hline Primary & $8314(55.4)$ & 45.4 \\
\hline Secondary & $6695(44.6)$ & 39.5 \\
\hline Male factor of infertility, $\mathrm{n}(\%)^{*}$ & $6193(41.3)$ & 44.9 \\
\hline Tubal factor of infertility, n (\%) & 8799 (58.6) & 42.2 \\
\hline $\begin{array}{l}\text { Anovulation factor of } \\
\text { infertility, } \mathrm{n}(\%)^{*}\end{array}$ & $1714(11.4)$ & 47.1 \\
\hline Unexplained infertility, n (\%) & $746(5.0)$ & 46.1 \\
\hline \multicolumn{3}{|l|}{ Treatment characteristics } \\
\hline Gn dosage, IU, mean $(S D)^{*}$ & $1920.99(1007.41)$ & \\
\hline Gn duration, days, mean $(S D)^{*}$ & $10.14(3.35)$ & \\
\hline \multicolumn{3}{|l|}{ Cycle protocols, n (\%) } \\
\hline Long agonist protocol & $9579(64.4)$ & 49.4 \\
\hline Short agonist protocol & $1176(7.9)$ & 28.9 \\
\hline Antagonist protocol & $1581(10.6)$ & 39.7 \\
\hline Minimal-stimulation protocol & $940(6.3)$ & 19.7 \\
\hline Ultra-long protocol & $434(2.9)$ & 52.3 \\
\hline Other protocol & $1153(7.8)$ & 23.9 \\
\hline \multicolumn{3}{|l|}{ Fertilization methods, n (\%) } \\
\hline IVF & $10,401(69.3)$ & 42.6 \\
\hline$|\mathrm{CS}|$ & $4074(27.2)$ & 43.7 \\
\hline Mixed IVF ICSI & $525(3.5)$ & 41.1 \\
\hline E2 on hCG day, pg/ml, mean (SD) ${ }^{*}$ & $3289.68(1915.22)$ & \\
\hline P on hCG day, ng/ml, mean (SD) & $1.21(18.29)$ & \\
\hline \multicolumn{3}{|l|}{ Year of transfer, $n(\%)^{*}$} \\
\hline 2013 & $3781(25.2)$ & 39.5 \\
\hline 2014 & $3894(25.9)$ & 41.7 \\
\hline 2015 & $3862(25.7)$ & 44.1 \\
\hline 2016 & $3475(23.1)$ & 46.2 \\
\hline \multicolumn{3}{|l|}{ Stage of embryos transferred, n (\%) } \\
\hline $\begin{array}{l}\text { Cleavage-stage embryo } \\
\text { (D2/D3/D4) }\end{array}$ & $14,236(94.8)$ & 42.8 \\
\hline Blastocyst (D5/D6/D7) & $776(5.2)$ & 43.9 \\
\hline
\end{tabular}

Table 1 Basic characteristics of the patients undergoing IVF/ICSI treatment (Continued)

\begin{tabular}{lll}
\hline Characteristics & $\begin{array}{l}\text { All cycles } \\
(\boldsymbol{n}=15,012)\end{array}$ & $\begin{array}{l}\text { LBR per } \\
\text { cycle (\%) }\end{array}$ \\
\hline Number of embryos transferred, $\mathrm{n}(\%)^{*}$ & & \\
1 & $4254(28.3)$ & 29.1 \\
2 & $10,758(71.7)$ & 48.2 \\
Oocytes retrieved, mean (SD) & $8.17(4.91)$ & \\
\hline
\end{tabular}

Abbreviations: $L B R$ Live-birth rate, $B M I$ Body mass index, SD Standard deviation ${ }^{*} P<0.05$ for comparisons between live-birth group and no live birth group, chi-square test was used for categorical variables, and t-test or Kruskal-Wallis test for continuous variables

results were found in the stratification analysis for cleavage-stage ET cycles although test for multiplicative interaction was not significant $(P=0.195)$.

\section{Discussion}

In the current study, we confirmed the nonlinearity in EMT-LBR association and a plateau of LBR per cycle when EMT was $11 \mathrm{~mm}$ or thicker. The embryo quality graded by cumulative score was significantly associated with LBR for cleavage-stage and for blastocyst ETs, and these associations were independent of the number of embryo transferred and EMT. To the best of our knowledge, this is the first multicenter retrospective cohort study to evaluate the independent and interaction effects of EMT on hCG day and embryo quality on the outcome of fresh IVF/ICSI cycles.

Our results are consistent with the reported association of thin EMT with poor pregnancy outcomes in fresh ET cycles $[4,7,12]$, while a consensus is still lacking on what the precise definition of thin endometrium. The present study showed EMT above $11 \mathrm{~mm}$ is related to a higher chance of delivery. This finding indicates that adequate endometrial development is favorable for improving chance of live-birth, which concurs to some extent with one recent study [12]. As discussed in the meta-analysis, a thickness threshold of $7 \mathrm{~mm}$ was frequently reported below which pregnancy rates decreased rapidly, but the case of EMT below $7 \mathrm{~mm}$ occurred infrequently [6] and the threshold was not available for live-birth evaluation. Therefore, there may be of more clinical significance for the EMT cut-off value of $11 \mathrm{~mm}$, which could be more helpful for decision making on fresh ET or freezing of embryos for potentially better chances to conceive with an endometrium developed under natural conditions in subsequent cryo-cycles. However, some others could not establish a significant correlation between EMT and the chance to conceive in the study population with euploid ETs [26]. Debate on the predictive value EMT in clinical outcomes is ongoing [6]. In order to investigate the real endometrium's effect, it is interesting to investigate the value of EMT in 


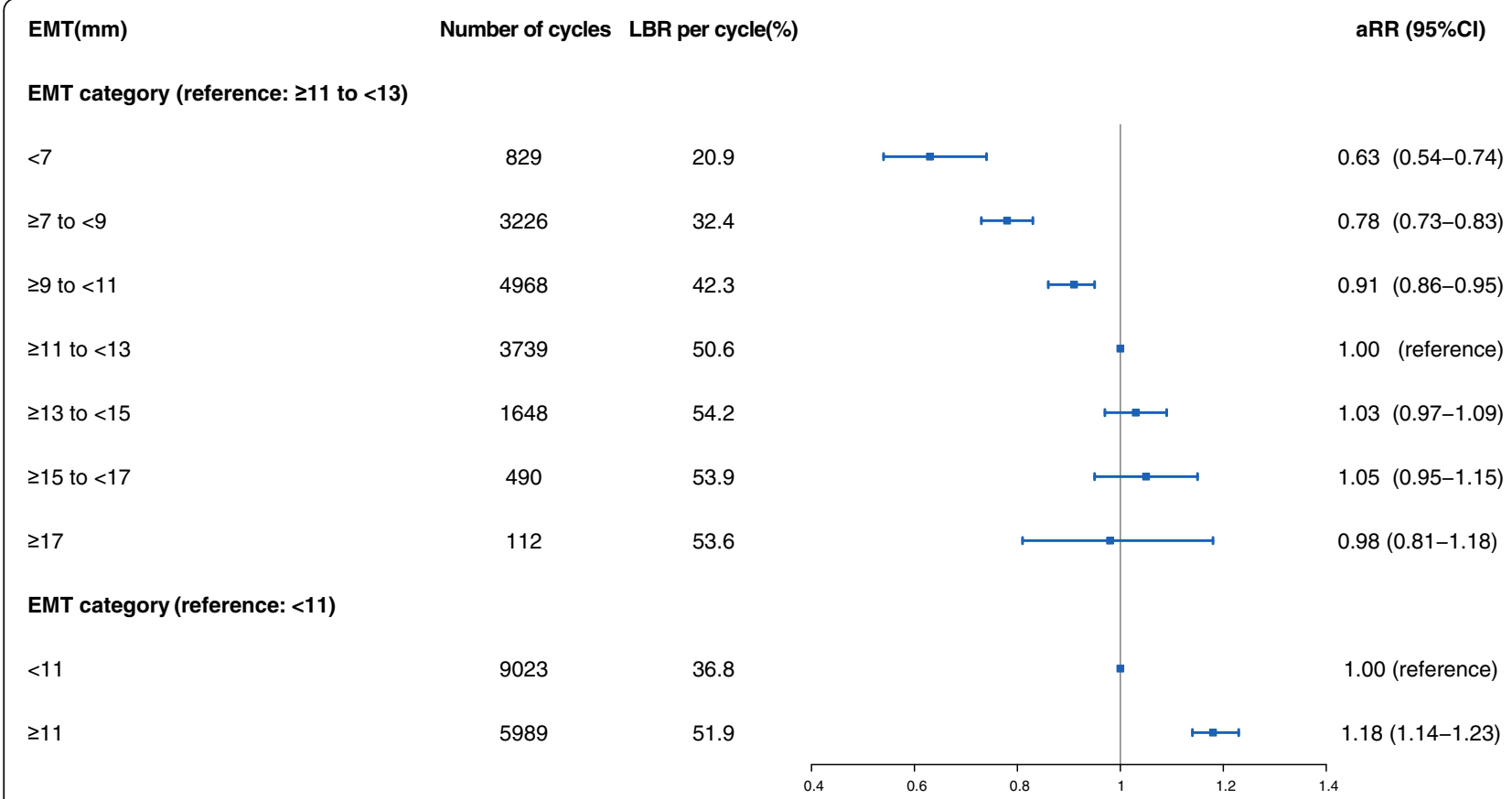

Fig. 2 Adjusted relative risk for live-birth according to EMT categories. Analysis Adjusted for maternal age, maternal BMI, basal FSH, basal LH, basal T, basal PRL, duration of infertility, infertility type, male factor of infertility, anovulation factor of infertility, Gn dosage, Gn duration, cycle protocols, E2 on hCG day, year of transfer, oocytes retrieved, location of fertility centers, number of embryos transferred and embryo score. Abbreviations: LBR, live-birth rate; aRR, adjusted relative risk; Cl, confidence interval

patients undergoing frozen embryo transfer cycles in the further study because in this population the possible negative effect of ovarian hyperstimulation on endometrial development is absent.

The mechanism of the association between thin endometrium and difficulty for implantation and development remains elusive. One speculation relates to that the implanting embryo would be much closer to the basal layer endometrium with higher oxygen concentrations in patients with thin functional layer EMT [27]. It is well known that high oxygen tensions could be detrimental for embryo implantation and development due to the production of reactive oxygen species [28]. In addition, it has been speculated that low late-follicular estradiol levels could result in inadequate endometrial proliferation [7, 29]. Follicular estrogen production is a reflection of follicular maturity and impacts both endometrial receptivity and follicular growth, oocyte maturation, sperm transport, embryo survival [30]. In other words, it is possible that the observed reduction in pregnancy rates associated with a thin endometrium may

Table 2 Adjusted relative risk for live-birth according to cleavage-stage embryo quality

\begin{tabular}{|c|c|c|c|c|c|}
\hline Embryo quality & Number of cycles & Number of live-birth (LBR per cycle, \%) & $\operatorname{aRR}(95 \% \mathrm{Cl})^{\mathbf{a}}$ & $\operatorname{aRR}(95 \% \mathrm{Cl})^{\mathbf{b}}$ & $\operatorname{aRR}(95 \% \mathrm{Cl})^{\mathbf{c}}$ \\
\hline Q1 (1 point) & 770 & 104 (13.5) & 1.00 (reference) & 1.00 (reference) & 1.00 (reference) \\
\hline Q2 (2 points) & 2620 & 802 (30.6) & $1.99(1.62-2.44)$ & $1.94(1.57-2.39)$ & $1.93(1.56-2.38$ \\
\hline Q3 (3 points) & 3315 & $1348(40.7)$ & $2.43(1.98-2.98)$ & $2.35(1.91-2.90)$ & $2.34(1.89-2.88)$ \\
\hline Q4 (4 points) & 3154 & $1590(50.4)$ & $2.88(2.35-3.53)$ & $2.74(2.21-3.40)$ & $2.72(2.19-3.38)$ \\
\hline Q5 (5 points) & 2993 & $1629(54.4)$ & $3.07(2.50-3.76)$ & $2.92(2.35-3.62)$ & $2.90(2.33-3.60)$ \\
\hline Q6 (6 points) & 1384 & $613(44.3)$ & $3.27(2.64-4.06)$ & $3.12(2.48-3.91)$ & $3.10(2.47-3.89$ \\
\hline$P_{\text {for trend }}$ & & & $<0.001$ & $<0.001$ & $<0.001$ \\
\hline
\end{tabular}

Abbreviations: $L B R$ Live-birth rate, aRR Adjusted relative risk, $C I$ Confidence interval

a Adjusted for maternal age, maternal BMI, Basal FSH, Basal LH, Basal T, Basal PRL, duration of infertility, infertility type, male factor of infertility, anovulation factor of infertility, Gn dosage, Gn duration, cycle protocols, E2 on hCG day, year of transfer, oocytes retrieved and location of fertility centers

${ }^{\mathrm{b}}$ Adjusted for maternal age, maternal BMI, basal FSH, basal LH, basal T, basal PRL, duration of infertility, infertility type, male factor of infertility, anovulation factor of infertility, Gn dosage, Gn duration, cycle protocols, E2 on hCG day, year of transfer, oocytes retrieved, location of fertility centers and number of embryos transferred

'Adjusted for maternal age, maternal BMI, basal FSH, basal LH, basal T, basal PRL, duration of infertility, infertility type, male factor of infertility, anovulation factor of infertility, Gn dosage, Gn duration, cycle protocols, E2 on hCG day, year of transfer, oocytes retrieved, location of fertility centers, number of embryos transferred and EMT 
Table 3 Adjusted relative risk for live-birth according to cleavage-stage embryo quality stratified by EMT, and EMT stratified by cleavage-stage embryo quality

\begin{tabular}{|c|c|c|c|c|c|c|}
\hline EMT & Q1 $(\boldsymbol{n}=770)$ & Q2 (n=2620) & Q3 (n=3315) & Q4 (n=3154) & Q5 (n=2993) & Q6 $(\boldsymbol{n}=1384)$ \\
\hline \multicolumn{7}{|l|}{$<11 \mathrm{~mm}$} \\
\hline $\begin{array}{l}\text { N live-births/N cycles } \\
\text { (LBR per cycle, \%) }\end{array}$ & 68/574 (11.8) & $457 / 1775$ (25.7) & 759/2104 (36.1) & $754 / 1702(44.3)$ & $803 / 1642(48.9)$ & $318 / 796$ (39.9) \\
\hline $\operatorname{aRR}(95 \% \mathrm{Cl})^{\mathrm{a}}$ & 1.00 (reference) & $1.86(1.43-2.41)$ & $2.38(1.84-3.09)$ & $2.85(2.17-3.75)$ & $3.13(2.38-4.12)$ & $3.67(2.74-4.91)$ \\
\hline \multicolumn{7}{|l|}{$\geq 11 \mathrm{~mm}$} \\
\hline $\begin{array}{l}\text { N live-births/N cycles } \\
\text { (LBR per cycle, \%) }\end{array}$ & $36 / 196(18.4)$ & $345 / 845(40.8)$ & $589 / 1211$ (48.6) & $836 / 1452(57.6)$ & $826 / 1351$ (61.1) & 295/588 (50.2) \\
\hline $\operatorname{aRR}(95 \% \mathrm{Cl})^{\mathrm{a}}$ & 1.00 (reference) & $2.05(1.43-2.94)$ & $2.35(1.64-3.37)$ & $2.68(1.86-3.86)$ & $2.78(1.93-4.00)$ & $2.75(1.89-4.01)$ \\
\hline $\operatorname{aRR}(95 \% \mathrm{Cl})^{b}$ & $0.95(0.61-1.46)$ & $1.23(1.09-1.40)$ & $1.18(1.07-1.29)$ & $1.22(1.13-1.32)$ & $1.17(1.08-1.26)$ & $1.12(0.98-1.27)$ \\
\hline
\end{tabular}

Abbreviations: $N$ Number, $L B R$ Live-birth rate, aRR Adjusted relative risk, $C$ C Confidence interval

a Adjusted for maternal age, maternal BMI, basal FSH, basal LH, basal T, basal PRL, duration of infertility, infertility type, male factor of infertility, anovulation factor of infertility, Gn dosage, Gn duration, cycle protocols, E2 on hCG day, year of transfer, oocytes retrieved, location of fertility centers and number of embryos transferred. Reference was Q1 group

${ }^{\mathrm{b}}$ Adjusted for maternal age, maternal BMI, basal FSH, basal LH, basal T, basal PRL, duration of infertility, infertility type, male factor of infertility, anovulation factor of infertility, Gn dosage, Gn duration, cycle protocols, E2 on hCG day, year of transfer, oocytes retrieved, location of fertility centers and number of embryos transferred. Reference was EMT $<11 \mathrm{~mm}$ group

be a consequence of another unmeasured aspect of oocyte/ embryo quality besides their morphologic grading. Furthermore, it is plausible that thin endometrium may be related to iatrogenic event like Asherman's syndrome [31] or use of clomiphene citrate, leading to an E receptor abnormality [27] and the subsequent lack of a normal proliferative response to the rising E level.

The thickened endometrium provides a site for attachment, as well as the source of nourishment for an implanting embryo during its first few weeks, until the placenta starts to develop. Whether a thick endometrium has a detrimental effect on clinical outcome is controversial [12, 13]. Our findings indicated that thick EMT $(>17 \mathrm{~mm})$ neither played an adverse role nor conferred additional benefit, similar to results reported in recent studies [12, 32], which suggested a ceiling effect of EMT after excluding patients with intrauterine pathologies such as polyps or fibroids.

Owing to the adverse perinatal risks and multiple pregnancy related costs, increased use of elective singleembryo transfer (eSET) in couples attempting assisted conception has been proposed and gradually adopted by some countries, such as Sweden and Belgium [22]. In other countries, however, the adoption of eSET in practice is hampered due to the desire of both clinicians and patients to ensure pregnancy and to reduce the costs of multiple ART cycles, especially in countries where ART is not publicly funded [33]. Opinion should, therefore, shift towards the view that the decision to transfer one or two embryos should be made according to not only the number but quality of available embryos. With regard to single embryo quality, there was a robust correlation between morphologic parameters and live-birth in previous studies transferring one cleavage-stage embryo [34] or one blastocyst [35]. In general, our findings support the validity of morphologic grading to be used to guide the embryo selection.

In addition, we also have realized that the association of live birth with embryo quality of different embryo combinations is poorly understood, while it is imperative for decision-making of optimal embryos number and quality to transfer. CES and mean embryo score (MES) based on 4-point embryo score have been proposed to predict pregnancy outcomes restricted to cleavage-stage embryos transfer $[17,36]$. Our data provided adequate support for the efficiency of CES (Poor $=1$ point, Fair $=2$ points, Good $=3$ points) in predicting live-birth when restricted to cleavage-stage ETs. For blastocyst transfer, it is also appropriate to determine the group of blastocyst embryo quality by cumulative score although we observed that a good-quality blastocyst transfer had similar LBR compared to that of a fair-quality blastocyst and combinations of blastocyst which was graded 'Poor' or 'Fair', which may be potentially caused by the limited cycles. To reduce the risk of multiple gestations, eSET was recommended to women with both one fair or good embryo and embryo pairs with the same cumulative score. Our findings doubted about other embryo scoring methods based on the number of good-quality embryos transferred [15] and grading of best embryo transferred [37] applied to distinguish different embryo combinations and to predict live-birth. Consequently, our findings filled the gaps of current embryo scoring systems and could help clinicians and infertile couples make a better choice.

It is estimated that embryos account for one-third of implantation failures, while suboptimal endometrial receptivity and altered embryo-endometrial dialogue are responsible for the remaining two-thirds [38]. A twoway communication between the embryo and receptive 
endometrium leads to implantation and the course of pregnancy, but ethical restrictions and the lack of mechanistic studies have delayed studies on embryoendometrial interactions in humans [39]. Endometrial receptivity and selectivity are two complementary concepts introduced to describe the endometrium, the emerging concept as a biosensor of embryo quality [40]. Either impaired embryo development potential or impaired endometrial selectivity/receptivity has a negative effect on the embryo-endometrial cross-talk. It is gradually acknowledged that the thickness of the endometrium can be used as a noninvasive parameter to infer on endometrial receptivity. Therefore, the investigation of interaction between embryo quality and EMT would provide some evidence. Our research showed that LBR could not be improved by thicker EMT when only one poor cleavage-stage embryo is available for transfer. In this circumstance, it is recommendable to restart the treatment for better embryo quality. What's more, the LBR did not increase significantly with thicker EMT with transfer of two good-quality cleavage-stage embryos and blastocysts in groups of embryo quality except in Q1 group. Our findings suggested that the requirement for optimal endometrial environment is less stringent when high quality embryos are available for transfer. It coincides with a previous study with a smaller sample size [29]. It seemed that the additional 2-3 days delay may provide an inadequate endometrium with additional time to develop. Taken together, the significant embryoendometrial interaction on live-birth suggests that the combination of EMT and embryos quality may improve the prognostic value in clinical practice for live-birth in patients undergoing transfer of 1-2 fresh cleavage-stage embryos, and for blastocyst transfers, embryo quality may be more important for treatment outcome compared with EMT.

Our findings were strengthened by 2-mm EMT-LBR relationship evaluation in consideration of clinical value in practice [7], in addition to establish a cutoff value of $11 \mathrm{~mm}$ for endometrial thickness above which LBR per cycle reached a plateau. Relying on a large sample size, transfers of different combinations of embryos were first compared with a good-quality ET to predict live-birth in detail, which confirmed the efficiency of CES separately for both cleavage-stage embryos and blastocysts and might compensate for the deficiency of some embryo scoring methods and improve embryo selection. It is noteworthy that the independent effects were confirmed in a prediction model with analysis of 100 prospectively recorded variables [4], while this was the first time to estimate the interaction of endometrial characteristics and embryo quality on LBR of fresh IVF/ICSI cycles. Still, the weaknesses of this study included its retrospective nature of the data, the possibility of unidentified confounding variables which could not be excluded, and the reproducibility of EMT and embryo grade assessment of different observers in different centers. Moreover, due to only a few blastocyst transfer cycles were conducted before 2016 (only 5.2\% included in this study), these findings of blastocyst transfer required verification in future studies based on a larger sample. The observations in our study were based on fresh cycles, so the conclusion may not apply to frozen cycles.

\section{Conclusions}

In conclusion, this study demonstrated the nonlinearity in EMT-LBR association and the EMT cut-off value of $11 \mathrm{~mm}$ which might be of more clinical significance for predicting live-birth. Embryo quality represented by $\mathrm{cu}-$ mulative score was an independently prognostic tool for LBR. The significant embryo-endometrial interaction on live-birth suggested the combination of EMT and embryo quality might be useful in making prognostications about the potential for LBR in patients undergoing transfer of 1-2 fresh cleavage-stage embryos in view of a number of important and potential confounding variables. For blastocyst transfers, embryo quality might be more important for treatment outcome when compared with EMT.

\section{Supplementary information}

Supplementary information accompanies this paper at https://doi.org/10. 1186/s12958-020-00636-6.

Additional file 1: Table S1. Adjusted relative risk for live-birth according to embryo combinations. Table S2. Adjusted relative risk for livebirth according to blastocyst embryo quality. Table S3. Adjusted relative risk for live-birth according to blastocyst embryo quality stratified by EMT, and EMT stratified by blastocyst embryo quality. Table S4. Adjusted relative risk for live-birth according to embryo quality stratified by EMT, and EMT stratified by embryo quality in long agonist protocol.

\section{Abbreviations}

ART: Assisted reproductive technology; EMT: Endometrial thickness; ET: Embryo transfer; RRs: Relative risks; Cls: Confidence intervals; LBR: Livebirth rate; IVF: In vitro fertilization; ICSI: Intracytoplasmic sperm injection; hCG: Human chorionic gonadotropin; CES: Cumulative embryo score; PGD: Preimplantation genetic diagnosis; PGS: Preimplantation genetic screening; IVM: In vitro maturation; BMI: Body mass index; FSH: Follicle stimulating hormone; LH: Luteinizing hormone; T: Testosterone; PRL: Prolactin; Gn: Gonadotropin; E2: Estradiol; P: Progesterone; $\mathrm{COH}$ : Controlled ovarian hyperstimulation; eSET: Elective single-embryo transfer; MES: Mean embryo score

\section{Acknowledgments}

The authors thank all of medical staffs in the First Affiliated Hospital of NMU, NMU Affiliated Maternity Hospital and Henan Maternal and Child Health Hospital for recording all the data in this study through the years.

\section{Authors' contributions}

$\mathrm{HL}, \mathrm{XZL}$ and JBD contributed to the conception and design. $\mathrm{HL}$ contributed to statistical analyzing and article writing. JBD contributed to revising the article. XFL, FYD, QL, SYT, LH, SYC, XMH, KZ, BX and XYL contributed to data collection. HXM, YKX, HBS, ZBH, GFJ, YCG and XRW contributed to revising 
the article critically for important intellectual content. The authors read and approved the final version of the manuscript.

\section{Funding}

Supported by National Key Research \& Development Program

[2016YFC1000200, 2018YFC1004200] and the State Key Program of National

Natural Science Foundation of China [31530047, 81830100, 81730041].

\section{Availability of data and materials}

The datasets used and analyzed during the current study are available from the corresponding author on reasonable request.

\section{Ethics approval and consent to participate}

This study was performed in accordance with guidelines outlined in the Declaration of Helsinki. All methods and information collection protocols were approved by the institutional review board of Nanjing Medical University, China NJMUIRB (2018) 009. Our research has obtained the waiver from institutional review board for the medical record review for selective variable analysis in three centers.

\section{Consent for publication}

Not applicable.

\section{Competing interests}

The authors declare that they have no competing interests.

\section{Author details}

'State Key Laboratory of Reproductive Medicine, Nanjing Medical University, Nanjing 211166, China. ²Department of Epidemiology, School of Public Health, Nanjing Medical University, Nanjing 211166, China. ${ }^{3}$ Department of Reproduction, the Affiliated Nanjing Maternity and Child Health Hospital of Nanjing Medical University, Nanjing 210004, China. ${ }^{4}$ Department of Reproduction, the First Affiliated Hospital with Nanjing Medical University, Nanjing 210029, China. ${ }^{5}$ Department of Reproduction, Henan Medical Maternity and Child Health Care Hospital, Henan 450052, China.

Received: 17 February 2020 Accepted: 29 July 2020

Published online: 21 August 2020

\section{References}

1. Sunderam S, Kissin DM, Crawford SB, Folger SG, Jamieson DJ, Warner L, et al. Assisted reproductive technology surveillance - United States, 2012 MMWR Surveill Summ. 2015:64:1-29.

2. Broer SL, van Disseldorp J, Broeze KA, Dolleman M, Opmeer BC, Bossuyt P, et al. Added value of ovarian reserve testing on patient characteristics in the prediction of ovarian response and ongoing pregnancy: an individual patient data approach. Hum Reprod Update. 2013;19:26-36.

3. Roseboom TJ, Vermeiden JP, Schoute E, Lens JW, Schats R. The probability of pregnancy after embryo transfer is affected by the age of the patient, cause of infertility, number of embryos transferred and the average morphology score, as revealed by multiple logistic regression analysis. Hum Reprod. 1995;10:3035-41.

4. Vaegter KK, Lakic TG, Olovsson M, Berglund L, Brodin T, Holte J. Which factors are most predictive for live birth after in vitro fertilization and intracytoplasmic sperm injection (IVF/ICSI) treatments? Analysis of 100 prospectively recorded variables in 8,400 IVF/ICSI single-embryo transfers. Fertil Steril. 2017;107:641-8 e642.

5. Richter KS, Bugge KR, Bromer JG, Levy MJ. Relationship between endometrial thickness and embryo implantation, based on 1,294 cycles of in vitro fertilization with transfer of two blastocyst-stage embryos. Fertil Steril. 2007:87:53-9.

6. Kasius A, Smit JG, Torrance HL, Eijkemans MJ, Mol BW, Opmeer BC, et al. Endometrial thickness and pregnancy rates after IVF: a systematic review and meta-analysis. Hum Reprod Update. 2014;20:530-41.

7. Ribeiro VC, Santos-Ribeiro S, De Munck N, Drakopoulos P, Polyzos NP, Schutyser $V$, et al. Should we continue to measure endometrial thickness in modern-day medicine? The effect on live birth rates and birth weight. Reprod Biomed Online. 2018;36:416-26.

8. Wu F, Liu F, Guan Y, Du J, Tan J, Lv H, et al. A nomogram predicting clinical pregnancy in the first fresh embryo transfer for women undergoing in vitro fertilization and intracytoplasmic sperm injection (IVF/ICSI) treatments. J Biomed Res. 2019:33:422-9.

9. Zhang T, He Y, Wang Y, Zhu Q, Yang J, Zhao X, et al. The role of threedimensional power Doppler ultrasound parameters measured on hCG day in the prediction of pregnancy during in vitro fertilization treatment. Eur J Obstet Gynecol Reprod Biol. 2016;203:66-71.

10. Seshadri S, Sunkara SK. Natural killer cells in female infertility and recurrent miscarriage: a systematic review and meta-analysis. Hum Reprod Update. 2014;20:429-38

11. Ruiz-Alonso M, Blesa D, Diaz-Gimeno P, Gomez E, Fernandez-Sanchez M, Carranza $F$, et al. The endometrial receptivity array for diagnosis and personalized embryo transfer as a treatment for patients with repeated implantation failure. Fertil Steril. 2013;100:818-24.

12. Yuan X, Saravelos SH, Wang Q, Xu Y, Li TC, Zhou C. Endometrial thickness as a predictor of pregnancy outcomes in 10787 fresh IVF-ICSI cycles. Reprod Biomed Online. 2016;33:197-205

13. Weissman A, Gotlieb L, Casper RF. The detrimental effect of increased endometrial thickness on implantation and pregnancy rates and outcome in an in vitro fertilization program. Fertil Steril. 1999;71:147-9.

14. Dietterich C, Check JH, Choe JK, Nazari A, Lurie D. Increased endometrial thickness on the day of human chorionic gonadotropin injection does not adversely affect pregnancy or implantation rates following in vitro fertilization-embryo transfer. Fertil Steril. 2002;77:781-6.

15. Cai Q, Wan F, Appleby D, Hu L, Zhang H. Quality of embryos transferred and progesterone levels are the most important predictors of live birth after fresh embryo transfer: a retrospective cohort study. J Assist Reprod Genet. 2014:31:185-94.

16. Laasch C, Puscheck E. Cumulative embryo score, not endometrial thickness, is best for pregnancy prediction in IVF. J Assist Reprod Genet. 2004;21:4750.

17. Terriou P, Sapin C, Giorgetti C, Hans E, Spach JL, Roulier R. Embryo score is a better predictor of pregnancy than the number of transferred embryos or female age. Fertil Steril. 2001;75:525-31.

18. Alpha Scientists in Reproductive M, Embryology ESIGo. The Istanbul consensus workshop on embryo assessment: proceedings of an expert meeting. Hum Reprod. 2011:26:1270-83.

19. Racowsky C, Vernon M, Mayer J, Ball GD, Behr B, Pomeroy KO, et al. Standardization of grading embryo morphology. Fertil Steril. 2010;94:11523.

20. Giorgetti C, Terriou P, Auquier P, Hans E, Spach JL, Salzmann J, et al. Embryo score to predict implantation after in-vitro fertilization: based on 957 single embryo transfers. Hum Reprod. 1995;10:2427-31.

21. Irani M, Reichman D, Robles A, Melnick A, Davis O, Zaninovic N, et al. Morphologic grading of euploid blastocysts influences implantation and ongoing pregnancy rates. Fertil Steril. 2017;107:664-70.

22. Lawlor DA, Nelson SM. Effect of age on decisions about the numbers of embryos to transfer in assisted conception: a prospective study. Lancet. 2012;379:521-7

23. Steer CV, Mills CL, Tan SL, Campbell S, Edwards RG. The cumulative embryo score: a predictive embryo scoring technique to select the optimal number of embryos to transfer in an in-vitro fertilization and embryo transfer programme. Hum Reprod. 1992;7:117-9.

24. Hu Y, Maxson WS, Hoffman DI, Ory SJ, Eager S, Dupre J, et al. Maximizing pregnancy rates and limiting higher-order multiple conceptions by determining the optimal number of embryos to transfer based on quality. Fertil Steril. 1998;69:650-7.

25. Hu L, Du J, Lv H, Zhao J, Chen M, Wang Y, et al. Influencing factors of pregnancy loss and survival probability of clinical pregnancies conceived through assisted reproductive technology. Reprod Biol Endocrinol. 2018;16:74.

26. Gingold JA, Lee JA, Rodriguez-Purata J, Whitehouse MC, Sandler B, Grunfeld $L$, et al. Endometrial pattern, but not endometrial thickness, affects implantation rates in euploid embryo transfers. Fertil Steril. 2015; 104:620-8 e625.

27. Casper RF. It's time to pay attention to the endometrium. Fertil Steril. 2011; 96:519-21.

28. Catt JW, Henman M. Toxic effects of oxygen on human embryo development. Hum Reprod. 2000;15(Suppl 2):199-206.

29. Zhang X, Chen CH, Confino E, Barnes R, Milad M, Kazer RR. Increased endometrial thickness is associated with improved treatment outcome for selected patients undergoing in vitro fertilization-embryo transfer. Fertil Steril. 2005;83:336-40. 
30. Pohler KG, Geary TW, Atkins JA, Perry GA, Jinks EM, Smith MF. Follicular determinants of pregnancy establishment and maintenance. Cell Tissue Res. 2012;349:649-64

31. Ruiz-Velasco V, Gonzalez Alfani G, Pliego Sanchez L, Alamillo VM. Endometrial pathology and infertility. Fertil Steril. 1997;67:687-92.

32. Chen SL, Wu FR, Luo C, Chen X, Shi XY, Zheng HY, et al. Combined analysis of endometrial thickness and pattern in predicting outcome of in vitro fertilization and embryo transfer: a retrospective cohort study. Reprod Biol Endocrinol. 2010;8:30.

33. Romundstad LB. Number of embryos to transfer: better safe than sorry? Lancet. 2012;379:497-8.

34. Racowsky C, Stern JE, Gibbons WE, Behr B, Pomeroy KO, Biggers JD. National collection of embryo morphology data into Society for Assisted Reproductive Technology Clinic Outcomes Reporting System: associations among day 3 cell number, fragmentation and blastomere asymmetry, and live birth rate. Fertil Steril. 2011;95:1985-9.

35. Heitmann RJ, Hill MJ, Richter KS, DeCherney AH, Widra EA. The simplified SART embryo scoring system is highly correlated to implantation and live birth in single blastocyst transfers. J Assist Reprod Genet. 2013;30:563-7.

36. Lee TH, Chen CD, Tsai YY, Chang LJ, Ho HN, Yang YS. Embryo quality is more important for younger women whereas age is more important for older women with regard to in vitro fertilization outcome and multiple pregnancy. Fertil Steril. 2006:86:64-9.

37. Montagut M, Santos-Ribeiro S, De Vos M, Polyzos NP, Drakopoulos P, Mackens $S$, et al. Frozen-thawed embryo transfers in natural cycles with spontaneous or induced ovulation: the search for the best protocol continues. Hum Reprod. 2016;31:2803-10.

38. Craciunas L, Gallos I, Chu J, Bourne T, Quenby S, Brosens JJ, et al. Conventional and modern markers of endometrial receptivity: a systematic review and meta-analysis. Hum Reprod Update. 2019;25:202-23.

39. Diedrich K, Fauser BC, Devroey P, Griesinger G. Evian annual reproduction workshop $\mathrm{G}$. the role of the endometrium and embryo in human implantation. Hum Reprod Update. 2007:13:365-77.

40. Macklon NS, Brosens JJ. The human endometrium as a sensor of embryo quality. Biol Reprod. 2014;91:98.

\section{Publisher's Note}

Springer Nature remains neutral with regard to jurisdictional claims in published maps and institutional affiliations.

Ready to submit your research? Choose BMC and benefit from:

- fast, convenient online submission

- thorough peer review by experienced researchers in your field

- rapid publication on acceptance

- support for research data, including large and complex data types

- gold Open Access which fosters wider collaboration and increased citations

- maximum visibility for your research: over $100 \mathrm{M}$ website views per year

At $\mathrm{BMC}$, research is always in progress.

Learn more biomedcentral.com/submissions 